\title{
Recuperación y representación del conocimiento experto del profesorado emérito de las universidades españolas: ingeniería del conocimiento para la reconstrucción de la misión de la universidad y la calidad de la docencia universitaria en el marco del proceso de convergencia europeo
}

Entidad que Financia: Plan Nacional de I+D+I. Ministerio de Ciencia e Innovación

Duración: 2009-2011 (3 años)

El proyecto de esta investigación se centra en tres aspectos fundamentales:

a) El conocimiento y la experiencia son bienes valiosos en nuestra sociedad. Bienes que cuesta mucho construir y conviene, por tanto, proteger y aprovechar;

b) Las universidades han sido, tradicionalmente, lugares especializados en la creación y sistematización de conocimiento avanzado en las diversas áreas científicas, incluido el conocimiento sobre los procesos de crear conocimiento y de transmitirlo a las futuras generaciones;

c) Por la amplitud de su experiencia y el valor de sus aportaciones, el profesorado emérito de las universidades constituye una valiosísima fuente de información sobre los procesos de crear, sistematizar y enseñar conocimientos a las generaciones de futuros profesionales y científicos.

Partiendo de ese triple punto de partida, la investigación se propone tres objetivos básicos: recuperar el conocimiento experto de los/as profesores eméritos españoles; sistematizar sus aportaciones individuales y colectivas a través de un sistema de representación que lo haga visible y útil; utilizar procedimientos de gestión del 
conocimiento y transferencia de tecnología para convertir los resultados de la investigación en material disponible tanto para gestores como profesores universitarios, para formadores de futuros profesores $y$, en general, para todos aquellos interesados en la institución universitaria y su mejora.

Utilizando diversas metodologías del "knowledge capturing" se pretende reconstruir la experiencia de los actuales profesores eméritos de las universidades españolas en su acción como docentes e investigadores. Cabe suponer que han superado los diversos filtros de acceso a la categoría de eméritos porque son personas con una notabilísima hoja de servicios a las universidades en las que trabajaron. Pretendemos explorar qué piensan los profesores eméritos sobre la universidad, su función social y su compromiso docente y científico; sobre qué ideas, creencias y percepciones fundamentaron su vida universitaria; cómo reconstruyen, con la capacidad de perspectiva que ahora poseen, lo que han sido su experiencia y sus aportaciones hasta llegar al actual nivel de excelencia que la institución universitaria les ha reconocido. Se podría indagar en los estilos de enseñanza y/o estilos profesionales (¿cómo "funcionan" profesionalmente?; si trabajan en grupo para la docencia, de qué manera, cómo proceden en los proyectos de investigación, cómo se relacionan con sus pares, vinculación institucional, etc...)

Recuperar el conocimiento experto del profesorado emérito exige, también, utilizar diversas técnicas de representación de dicho conocimiento. En nuestro caso planteamos la representación del conocimiento experto del profesorado emérito a través de esquemas conceptuales informatizados (en la línea de las modernas técnicas de ingeniería del conocimiento) que permitan una visión de conjunto y el establecer la relación existente entre elementos de las creencias y las prácticas de los diversos participantes. Las diversas modalidades de mapas conceptuales, ya empleadas por el equipo en proyectos anteriores nos servirán para sistematizar y establecer interacciones entre las diversas aportaciones de los sujetos estudiados y, también, para poder navegar entre sus diversos niveles de contenidos y evidencias.

Ocho universidades españolas (Santiago de Compostela, Granada, La Coruña, Navarra, Sevilla, Tarragona, Valencia y Vigo), en colaboración con tres extranjeras (Roma, Lisboa, Hamburgo) y un instituto de ingeniería del conocimiento (el Institute of Machine and Human Cognition, IHMC, de Pensacola, Florida vinculado a la Univ. de West Florida) estudiarán la visión de la universidad y de su función docente e investigadora de los 360 profesores/as universitarios eméritos.

Los análisis se realizarán a tres niveles complementarios: análisis intra-sujeto (para identificar las ideas y prácticas propias de cada uno de los docentes participantes); análisis por grupo de especialildad (para establecer los patrones básicos de la cultura de cada gran especialidad- humanidades, ciencias sociales y jurídicas, ciencias experimentales, ingenierías, ciencias de la salud, si la hubiere); análisis global del conjunto del profesorado emérito (para identificar los factores básicos que configuran el pensamiento y las prácticas, y la relación entre ambos, de quienes han sido docentes e investigadores universitarios relevantes). 
Finalmente, los resultados y las conclusiones de esta investigación ha de redundar en beneficio de la sociedad y de las instituciones universitarias y sus agentes. Por eso, todas las informaciones y resultados de la investigación se podrán a disposición de la sociedad generando un dispositivo virtual que permita acceder al conocimiento y experiencia recuperadas a través del proyecto y beneficiarse de ellas. Aparte del Informe Final, con los resultados de los análisis mencionados, el proyecto ofrecerá entre sus productos un portal WEB donde se recogerán las aportaciones estudiadas junto al análisis de los investigadores y las evidencias reunidas de forma tal que puedan servir como punto de referencia para quienes, a título personal o institucional, están comprometidos en la reconstrucción de la universidad española y europea.

\section{Bibliografía}

Allport, G. (1942): The use of Personal Documents in Psychological Science, en Social Science Research Council Bulletin, $\mathrm{n}^{\circ}$ 49. N.York.

Bain, K. (2006): Lo que hacen los mejores profesores universitarios. Valencia: Servei de publicacións, Universitat de Valencia.

Cooke, N.J. (1994): Varieties of Knowledge Elicitation Techniques. Internatinal Journal of Human-Computer Studies, 41, 801-849.

Evans, L. y Abbott, I. (1998): Teaching and Learning in Higher Education. London: Casell

Ferrarotti, F. (1983) : Histoire et histories de vie. Librairie des Meridiens. París.

Gow, L. \& Kember, D. (1993): Conceptions of Teaching and Their Relationship to Student Learning. British Journal or Educational Psychology, 63: 20-33.

Hativa, N. y Goodyear, P. (Edit, 2001): Teacher Thinking, Beliefs and Knowledge in Higher Education. Dordrecht: Kluwer Academic Publishers.

Kember, D. (2001): Beliefs about Knowledge and the process of Teaching and Learning as a Factor in Adjusting to Study in Higher Education. Studies in Higher Education, 26 (2), 205-221.

Kugel, P. (1993): How profesors develop as teachers. Studies in Higher Education, vol. 18 (3).

Novak, J.D. (1998): Conocimiento y aprendizaje. Los mapas conceptuales como herramientas facilitadoras para escuelas y empresas. Madrid: Alianza.

Pinar, W. (1981): Life history and Educational experience, en Journal of Curriculum Theorizing, 3(1) pag. 259-286.

Robertson, D.L (1999): Profesors'perspectives on their teaching: a new construct and developmental model. Innovative Higher Education, vol. 23(4), 271-294. 
Sugrue, C., Day, C. (Edits. 2001): Developing Teachers and Teaching: International Research Perspectives. London. Falmer Press.

Trigwell, K. \& Prosser, M. (1999): Understanding Learning and Teaching. Buckinghan: Open Univ. Press.

Zabalza Beraza, M.A. (1988): Pensamiento del profesor y desarrollo didáctico, Enseñanza, nº 4-5, Pg. 109-138.

Zabalza Beraza, M.A. (2003): Competencias docentes del profesorado universitario. Madrid. Narcea.

\section{Investigadores}

\section{Investigador principal:}

Dr. Miguel Ángel Zabalza Beraza, catedrático del Departamento de Didáctica y Organización Escolar de la Universidade de Santiago de Compostela.

\section{Investigadora coordinadora en la URV:}

Dra. Charo Barrios Arós, profesora titular de universidad del Departamento de Pedagogía de la Universitat Rovira i Virgili.

\section{Altres investigadors de la URV:}

Dr. Manuel Fandos Garrido, técnico del Instituto de Ciencias de la Educación de la Universitat Rovira i Virgili.

Dr. Ángel-Pío González Soto, catedrático de universidad del Departamento de Pedagogía de la Universitat Rovira i Virgili.

Dra. Pilar Iranzo García, profesora en comisión de servicios en el Departamento de Pedagogía de la Universitat Rovira i Virgili.

Dr. José Miguel Jimenéz González, profesor titular de universidad del Departamento de Pedagogía de la Universitat Rovira i Virgili.

Dra. Juana María Tierno García, profesora colaboradora permanente del Departamento de Pedagogía de la Universitat Rovira i Virgili. 